\title{
Integration im kommunalen Raum: Bremen und Newcastle-upon-Tyne im Vergleich
}

Gegensätzliche Entwicklungen scheinen die Einwanderungsgeschichte Großbritanniens und Westdeutschlands in der zweiten Hälfte des 20.Jahrhunderts zu prägen. Die Forschungsliteratur hat zum Teil sehr plakativ solche Unterschiede herausgearbeitet: deutsche Germanisierungspolitik versus britischen Multikulturalismus, befristeter Zugang von Arbeitskräften nach Westdeutschland versus offene Einwanderung nach Großbritannien oder deutsches nationalistisches Staatsangehörigkeitskonzept versus britische liberale Staatsbürgerschaft nach westeuropäischem Muster. ${ }^{1}$ Während Politik und Gesellschaft in Großbritannien akzeptiert hätten, dass das Land ein Einwanderungsland mit kultureller Vielfalt sei, habe die Bundesrepublik Deutschland über Jahrzehnte verneint, ein Einwanderungsland zu sein. ${ }^{2}$ Panikos Panayi zufolge lassen sich die unterschiedlichen politischen und gesellschaftlichen Strategien im Umgang mit Zuwanderung nur durch die Beobachtung von drei Schlüsselbereichen erschließen, die in den Jahrzehnten vor 1945 gründeten: 1. imperiale Traditionen, 2. langwährende Migrationsverhältnisse und Migrationsregime sowie 3. Staatsangehörigkeitsverhältnisse. ${ }^{3}$

Der hier angestrebte Vergleich der Einwandererbevölkerungen in Bremen und Newcastle-upon-Tyne muss solche weitreichenden Erörterungen über die Zuwanderungsgeschichte Deutschlands und Großbritanniens berücksichtigen, darf aber zugleich spezifische lokale Voraussetzungen und Bedingungen nicht vernachlässigen. Bremen und Newcastle sind zwei Städte, deren Einwanderungsgeschichte in der Forschung vernachlässigt wurde, die aber insofern Aufmerksamkeit verdienen, als in beiden Kommunen eine ausgeprägte regionale Identität auszumachen ist. Damit ist ein Element benannt, von dem zu erwarten steht, dass es Einfluss auf die Erfahrungen der jeweiligen Einwandererbevölkerung hatte.

Die Region um Newcastle rühmt sich ihrer Weltoffenheit und ihrer Gastfreundschaft. ${ }^{4}$ Für Bremen ist dagegen die Herausbildung einer spezifischen kommunalen Identität betont worden, die als Katalysator für Nationalismus und Xenophobie gewirkt habe. ${ }^{5}$ Die begrenzte Reichweite solcher Einschätzungen zeigen neuere Entwicklungen: Während Bremen in den PISA-Studien 2000 und 2003 sehr schlechte Ergebnisse in Bezug auf die Bildung der Einwandererkinder erzielte, verdeutlichen Initiativen wie der „Stadtplan der Religionen“ zu Beginn des 21. Jahrhunderts und die „Integrationswochen“ 2008 und 2010 das weitreichende

\footnotetext{
${ }^{1}$ Rogers Brubaker, Citizenship and Nationhood in France and Germany, Cambridge, MA 1992; Adrian Favell, Philosophies of Integration: Immigration and the Idea of Citizenship in France and Britain, Basingstoke 1998; Christian Joppke, Immigration and the Nation-State: The United States, Germany, and Great Britain, Oxford 1999; Karen Schönwälder, Einwanderung und ethnische Pluralität: politische Entscheidungen und öffentliche Debatten in Großbritannien und der Bundesrepublik von den 1950er bis zu den 1970er Jahren, Essen 2001; Leo Lucassen, The Immigrant Threat: The Integration of Old and New Migrants in Western Europe since 1850, Urbana/Chicago 2005.

${ }^{2}$ Panikos Panayi, The Evolution of Multiculturalism in Britain and Germany. A Historical Survey, in: Journal of Multilingual and Multicultural Development 25 (2004), H.5, S.466-480, hier S.472.

${ }^{3}$ Ebenda, S.468.

${ }^{4}$ Nigel Todd, Black on Tyne: The Black Presence on Tyneside in the 1860s, in: North-East Labour History Society Journal 21 (1987), S.17-27; David Renton, Colour Blind? Race and Migration in North East England since 1945, Sunderland 2007.

${ }^{5}$ Dieter K. Buse, Urban and National Identity: Bremen, 1860-1920, in: Journal of Social History 26 (1993), H.3, S.521-537.
} 
Engagement der Stadt für die Zuwandererbevölkerung und deren Integration. In Newcastle wird das jährliche „Mela-Festival“ als eine beliebte Veranstaltung herausgestellt, die darauf zielt, asiatische Kultur, Musik und Künste zu fördern, während in der Stadt zugleich ein Anstieg an rassistischer Diskriminierung zu beobachten ist und immer mehr rassistisch geprägte Vorfälle verzeichnet werden. ${ }^{6}$

Dieser Beitrag, der einer ausführlichen Studie zum Gegenstand vorangeht ${ }^{7}$, strebt nach einem Überblick über Zuwanderung und Integration auf lokaler Ebene vor dem Hintergrund der Geschichte zweier grundlegend verschiedener Einwanderungsländer. ${ }^{8}$ Zunächst wird dazu die Anwerbung ausländischer Arbeitskräfte in Westdeutschland in den Blick genommen, um in der Folge nach den langfristigen Folgen des spezifischen westdeutschen Migrationsregimes für den lokalen Raum zu fragen. Im Mittelpunkt stehen dabei die Erfahrungen türkischer Zuwanderer und deren Integration im Arbeits-, Wohnungs- und Bildungsbereich. Diese Bereiche sind ausgewählt worden, weil von wissenschaftlicher Seite mit Daten des Sozio-oekonomischen Panels (SOEP) und der letzten PISA-Studie (Programme for International Student Assessment) auf erhebliche Probleme der Integration hingewiesen worden ist: relativ hohe Erwerbslosenquoten, niedrige Wohnqualität und schlechte Schulabschlüsse. ${ }^{9}$ Der Vergleich mit der südasiatischen Bevölkerung der Stadt Newcastle hat die Funktion, die für Bremen gewonnenen Erkenntnisse einzuordnen. ${ }^{10}$

\section{Erwerbstätigkeit}

Die Aufnahme von Zuwanderern auf dem Arbeitsmarkt bildete in der Vergangenheit immer einen zentralen Indikator für deren Integration. Ökonomische Erwägungen haben nicht nur viele Migranten dazu bewegt, nach dem Zweiten Weltkrieg nach Großbritannien und Deutschland zu kommen. Sie sind darüber hinaus ein zentrales Element, das Alltag und Erfahrungen der Migranten prägt. Wissenschaftliche Untersuchungen haben seit den 1960er Jahren hinsichtlich der Arbeitsmarktintegration von Zuwanderern in beiden Ländern

\footnotetext{
${ }^{6}$ Anoop Nayak, Race, Place and Globalization: Youth Cultures in a Changing World, Oxford 2003, S. $44 \mathrm{f}$.

${ }^{7}$ Sarah E. Hackett, Foreigners, Minorities, Integration: The Muslim Immigrant Experience in Britain \& Germany, Manchester [2012].

${ }^{8}$ Den Angaben des Office for National Statistics (ONS) zufolge ist die indische, pakistanische und bangladeschische Bevölkerung Newcastles zwischen 1961 und 2001 von 1202 auf 5704 angewachsen. Es gilt allerdings zu berücksichtigen, dass diese Zahlen lediglich die Personen einschließen, die in Indien, Pakistan und Bangladesch geboren wurden, nicht jedoch deren Nachkommen. Der tatsächliche Umfang der Einwandererbevölkerung war daher zweifelsohne schon immer deutlich höher. Gemäß den Angaben des Statistischen Landesamtes Bremen lebten im Jahr 2009 insgesamt 36406 Menschen türkischer Herkunft in Bremen.

${ }^{9}$ Andreas Ammermüller, Poor Background or Low Returns? Why Immigrant Students in Germany Perform so Poorly in the Programme for International Student Assessment, in: Education Economics 15 (2007), H.2, S.215-230; Peter Dörschler/Pamela Irving Jackson, Host Nation Language Ability and Immigrant Integration in Germany: Use of GSOEP to Examine Language as an Integration Criterion, in: Democracy and Security 6 (2010), H.2, S. 147-182.

${ }^{10}$ Sarah E. Hackett, The Asian of the North: Immigrant Experiences and the Importance of Regional Identity in Newcastle upon Tyne during the 1980s, in: Northern History 46 (2009), H.2, S.293-311; dies., Peering Around the ,Velvet Curtain of Culture': The Employment and Housing of Newcastleupon-Tyne's Muslim Immigrants, 1960s-1990s, in: Gerald MacLean (Hrsg.), Britain and the Muslim World: Historical Perspectives, Newcastle-upon-Tyne 2011, S.222-237; dies., A Learning Curve: The Education of Immigrants in Newcastle-upon-Tyne and Bremen from the 1960s to the 1980s, in: Zvi Bekerman/Thomas Geisen (Hrsg.), International Handbook of Migration, Minorities, and Education Understanding Cultural and Social Differences in Processes of Learning, Dordrecht 2011, S.349-364.
} 
Aspekte wie Erwerbslosigkeit, rassistische Diskriminierung und geringes Qualifikationsniveau hervorgehoben. ${ }^{11}$ Nichtsdestoweniger lassen sich grundlegende und tief verankerte Unterschiede zwischen Großbritannien und der Bundesrepublik Deutschland im Blick auf die Grundlagen der ökonomischen Integration ausmachen: Die „Gastarbeiter“ sollten in Westdeutschland lediglich der temporären wirtschaftlichen Entlastung dienen. Die Anwerbung über die Bundesanstalt für Arbeit, die ausschließliche Orientierung an der Arbeitsfähigkeit der Zuwanderer und die zeitliche Befristung der Arbeitsverträge verdeutlichen das. ${ }^{12}$ Und viele Erfahrungsberichte, wie zum Beispiel in Güney Dals „Wenn Ali die Glocken läuten hört“ und Günter Wallraffs bahnbrechendem Werk „Ganz unten“, haben eindrücklich die Erfahrung der Migranten geschildert, ausschließlich als temporäre Arbeitskräfte zur Schließung von Lücken am Arbeitsmarkt gesehen zu werden ${ }^{13}$ - den Alltag von Isolierung, Orientierungslosigkeit, Vorurteilen und Rassismus.

Die lokale Situation in Bremen bildete keine Ausnahme: In der Stadt war eine Handvoll Unternehmen angesiedelt, die in den 1960er und frühen 1970er Jahren „Gastarbeiter“ anwarben. Dazu gehörten die beiden Schiffswerften AG Weser und Bremer Vulkan AG, die Bremer Woll-Kämmerei AG und der Klöckner Stahl- und Metallhandel. ${ }^{14}$ Die Unternehmen waren sich einig, dass die Beschäftigung ausländischer Arbeitskräfte befristeter Natur und der finanzielle Aufwand für ihre Aufnahme minimal bleiben sollte: Die „Gastarbeiter" wurden mit Vokabelheften ausgestattet, die ausschließlich Wörter und Redewendungen enthielten, die sie an ihrer Arbeitsstelle benötigten; einige Unternehmen senkten die Kosten, indem sie türkische Arbeitskräfte bereits in der Türkei einarbeiteten, anstatt bis zu ihrer Ankunft in Bremen zu warten. Sorgen galten der Frage, ob „Gastarbeiter“ bereits mit gesundheitlichen Beeinträchtigungen eintreffen könnten oder kurz nach ihrer Ankunft erkrankten und so die lokale Infrastruktur im Gesundheitswesen belasteten. ${ }^{15}$

Bei einem Vergleich mit Newcastle wird sichtbar, wie stark dieses System die ökonomische Integration der Zuwanderer behinderte. Wie in vielen anderen britischen Städten, hat sich die indische, pakistanische und bangladeschische Bevölkerung Newcastles im Laufe der Zeit vorzugsweise als Kleinunternehmer selbstständig gemacht. ${ }^{16}$ Ein Großteil dieser südasiatischen Einwanderer arbeitete als Handelsreisende, die an den Haustüren

\footnotetext{
${ }^{11}$ Christoph M. Schmidt, Immigrant Performance in Germany: Labor Earnings of Ethnic German Migrants and Foreign Guest-Workers, in: The Quarterly Review of Economics and Finance 37 (1997), Anhang 1, S.379-397; Panikos Panayi, Ethnic Minorities in Nineteenth and Twentieth-Century Germany: Jews, Gypsies, Poles, Turks and Others, London 2000, S.221-224; William W. Daniel, Racial Discrimination in England, Harmondsworth 1968; Tariq Modood, Employment, in: ders. u.a. (Hrsg.), Ethnic Minorities in Britain: Diversity and Disadvantage, London 1997, S.83-149.

${ }^{12}$ Jürgen Fijalkowski, Gastarbeiter als industrielle Reservearmee?, in: Archiv für Sozialgeschichte 24 (1984), S.399-456; Ulrich Herbert/Karin Hunn, Guest Workers and Policy on Guest Workers in the Federal Republic: From the Beginning of Recruitment in 1955 until its Halt in 1973, in: Hanna Schissler (Hrsg.), The Miracle Years: A Cultural History of West Germany, 1949-1968, Princeton 2001, S. 187-218.

${ }^{13}$ Güney Dal, Wenn Ali die Glocken läuten hört, Berlin 1979; Günter Wallraff, Ganz unten: Mit einer Dokumentation der Folgen, Köln 1988.

${ }^{14}$ Volkmar Leohold, Die Kämmeristen: Arbeitsleben auf der Bremer Woll-Kämmerei, Hamburg 1986; Diethelm Knauf/Helga Schröder, Fremde in Bremen: Auswanderer, Zuwanderer, Zwangsarbeiter, Bremen 1993; Hasan Çil, Anfänge einer Epoche, Berlin 2003.

${ }^{15}$ Staatsarchiv Bremen, 4,124/3-643 Sozialhilfe für Ausländer und Staatenlose - Allgemeines, Bd.1, 1964-1972; Staatsarchiv Bremen, 7,2121/1-711 Anwerbung, Vermittlung und Ausbildung ausländischer Arbeiter 1970-1973.

${ }^{16}$ Sheila Allen/Stuart Bentley/Joanna Bornat, Work, Race and Immigration, Bradford 1977; Modood, Employment, S.83; Commission on the Future of Multi-Ethnic Britain, The Future of Multi-Ethnic Britain: The Parekh Report, London 2000, S.201-204.
} 
Kleidungsstücke auf Kredit verkauften, oder sie betrieben Einzelhandelsgeschäfte. In beiden Fällen waren sie auf den Kontakt und die Interaktion mit der einheimischen Bevölkerung angewiesen. Von Bedeutung war dabei nicht nur, dass die Kunden der lokalen Arbeiterklasse angehörten, sondern auch, dass diese Handelsreisenden in einem Feld tätig waren, das unter der britischen Arbeitsbevölkerung, insbesondere der im Nordosten, nicht unüblich war und somit nicht als ein spezifisches „ethnic business“ galt. Des Weiteren ergaben sich für die Zuwanderer aus Indien, Pakistan und Bangladesch offenbar nur wenige Probleme bei dem Aufbau ihrer Unternehmen, sie nahmen keine finanzielle Unterstützung von außen oder Beratung für ihre unternehmerischen Vorhaben in Anspruch, sondern orientierten sich an erfolgreichen Unternehmensgründungen aus ihrem Umfeld. Viele dieser Männer waren nur so lange beispielsweise als Busfahrer oder Fabrikarbeiter tätig, bis sie genügend finanzielle Mittel für die Gründung eines Kleinunternehmens erwirtschaftet hatten. ${ }^{17}$

Berufliche Selbstständigkeit war unter Angehörigen der Zuwandererbevölkerung in Deutschland keineswegs ausgeschlossen. Das zeigt eine Vielzahl wissenschaftlicher Studien. ${ }^{18}$ Dennoch lassen sich erhebliche Unterschiede zwischen Newcastle und Bremen bzw. Großbritannien und Deutschland ausmachen. Erstens scheinen türkische Gewerbebetriebe in Bremen erst zu einem späteren Zeitpunkt entstanden zu sein als südasiatische Unternehmen in Newcastle. Während aus dem Ausland zugewanderte Unternehmer in Newcastle bereits in den 1980er Jahren ein weit verbreitetes Phänomen darstellten, traten sie in Bremen erst nach der Jahrtausendwende häufiger in Erscheinung. ${ }^{19}$ Die berufliche Selbstständigkeit für Angehörige ethnischer Minderheiten in Bremen konnte mithin aufgrund der anfänglichen Einschränkungen durch das „Gastarbeiter“-System erst später umgesetzt werden. Zweitens unterschieden sich die Unternehmen, die in Newcastle und Bremen von Zuwanderern aufgebaut wurden, in ihrer Anlage. Die Gewerbebetriebe in Newcastle, wie in ganz Großbritannien, konnten erfolgreich einheimische und ethnische kulturelle Muster innerhalb des etablierten Rahmens des britischen Einzelhandels zusammenführen. Als Beispiel dafür mag das in Birmingham entstandene „Balti“-Currygericht dienen, das für britische Kunden kreiert wurde. Für Deutschland wird dagegen nicht nur darauf hingewiesen, dass die Eröffnung von Döner-Schnellimbissen in den 1970er Jahren eine Möglichkeit bot, sich der Erwerbslosigkeit nach der Ölpreiskrise und einer Rückkehr in die Türkei zu entziehen, sondern dass türkische Zuwanderer sich seither vermehrt dazu gezwungen sahen, in andere Bereiche auszuweichen, da der Verkauf türkischen Essens sich als immer weniger

\footnotetext{
${ }^{17}$ Jon Gower Davies, The Evangelistic Bureaucrat. A Study of a Planning Exercise in Newcastle upon Tyne, London 1972; John H. Taylor, The Half-Way Generation: A Study of Asian Youths in Newcastle upon Tyne, Windsor 1976. Diese Vorgehensweise hat sich durch Archivrecherchen und Interviews bestätigt. Siehe Tyne and Wear Archives Service (TWAS), MD.NC/162/1, Local Government and Racial Equality Sub-Committee of Corporate Joint Sub-Committee, 18.3.1983-17.7.1985. Black Business Development Project; City of Newcastle upon Tyne Economic Development Committee. Ethnic Minority Groups \& Business Development, 4.3.1984; TWAS, MD.NC/614/2, Racial Equality Working Group, 18.1.1996-6.3.1997. LIA Newcastle, Minority Ethnic Businesses in Newcastle. Report of Survey Undertaken by Chief Executive's Department Newcastle City Council 1997. Zwischen Oktober 2004 und Juni 2010 wurden Interviews mit drei Generationen südasiatischer Einwanderer durchgeführt.

${ }^{18}$ Amelie Constant/Klaus F. Zimmermann, The Making of Entrepreneurs in Germany: Are Native Men and Immigrants Alike?, in: Small Business Economics 26 (2006), H.3, S.279-300; Maria Kontos, Self-Employment Policies and Migrants' Entrepreneurship in Germany, in: Entrepreneurship and Regional Development 15 (2003), H.2, S.119-135.

${ }^{19}$ Kleine Anfrage der Fraktion Bündnis 90/Die Grünen vom 21.4.2004. Wirtschaftsförderung für Unternehmer und Existenzgründer mit Migrationshintergrund. Antwort des Senats vom 25.5.2004, Drucksache 16/264, Bremische Bürgerschaft, Landtag, 16. Wahlperiode und Mitteilung des Senats vom 25.5.2004. Das wirtschaftliche Potenzial von Unternehmern und Existenzgründern mit Migrationshintergrund, Drucksache 16/262, 26.5. 2004, Bremische Bürgerschaft, Landtag, 16. Wahlperiode.
} 
rentabel herausstellte. ${ }^{20}$ Innerhalb dieses Schemas stellte Bremen gewiss keine Ausnahme dar. $^{21}$

Drittens gibt es grundlegende Unterschiede hinsichtlich der Frage, warum südasiatische beziehungsweise türkische Unternehmen in Newcastle und Bremen gegründet wurden und wie sie geführt werden. Interviews mit Zeitzeugen belegen, dass viele türkische Unternehmer in Bremen sich aufgrund von Diskriminierungserfahrungen bei der Arbeitsuche für die Selbstständigkeit entschieden. ${ }^{22}$ Obwohl in der Literatur vielfach behauptet wird, dass dies lange Zeit auch in Großbritannien der Fall war, haben Interviewte in Newcastle nicht davon berichtet. ${ }^{23}$ Zudem hat es den Anschein, dass rassistische und religiöse Diskriminierungen oft mit darüber entschieden, wie die türkischen Unternehmen in Bremen geführt wurden. Einige der Interviewten lehnen es beispielsweise ab, türkische Frauen einzustellen, die ein Kopftuch tragen, oder sie beschränken sich ausschließlich auf den Verkauf hochwertiger Produkte und versuchen auf diese Weise ein größeres deutsches Klientel aufzubauen. Türkische Unternehmer in Bremen mussten sich somit weitgehend von ihrer eigenen Bevölkerungsgruppe und Kultur lösen, um ökonomischen Erfolg zu erzielen.

\section{Der Wohnungsmarkt}

Untersuchungen über die Wohnsituation von Zuwanderern in Deutschland betonen, dass diese von der Regierungspolitik vernachlässigt und bei der Vergabe von Sozialwohnungen benachteiligt worden seien, Vermieter sie diskriminierten und sie sich insgesamt mit einer anderen Wohnsituation konfrontiert sahen als die einheimische Bevölkerung. ${ }^{24}$ Studien zu Großbritannien heben die ethnische Segregation und den Wohnungsmangel hervor. ${ }^{25}$ Ausmachen lässt sich, dass viele Probleme hinsichtlich der Entwicklung der Wohnsituation von Zuwanderern in beiden Ländern gleichermaßen beobachtet werden können. Aber auch in diesem Feld hat die spezifische Situation der befristeten Anwerbung von „Gastarbeitern" in der Bundesrepublik langfristige Folgen, die zu klaren Unterschieden in der Wohnsituation zwischen Bremen und Newcastle führten. Erstens waren „Gastarbeiter“ bei

\footnotetext{
${ }^{20}$ Joyce Marie Mushaben, Thinking Globally, Integrating Locally: Gender, Entrepreneurship and Urban Citizenship in Germany, in: Citizenship Studies 10 (2006), H.2, S.215; Roger Boyes/Dorte Huneke, Is it Easier to be a Turk in Berlin or a Pakistani in Bradford?, London 2004, S.21.

${ }^{21}$ Antrag der Fraktion Bündnis 90/Die Grünen. Stärkung von Unternehmen und Unternehmensgründungen mit migrantischem Hintergrund, Drucksache 16/810, 29.11.2005, Bremische Bürgerschaft, Landtag, 16. Wahlperiode.

${ }^{22}$ Interviews mit türkischen Einwanderern, die Erfahrungen mit der Selbstständigkeit gesammelt haben, wurden zwischen Januar 2006 und August 2010 durchgeführt.

${ }^{23}$ Susan Nowikowski, Snakes and Ladders: Asian Businesses in Britain, in: Robin Ward/Richard Jenkins (Hrsg.), Ethnic Communities in Business: Strategies for Economic Survival, Cambridge 1984, S.149-165; Gozde Inal, Why Do Minorities Launch Businesses in Britain?, in: International Journal of Business and Globalisation 1 (2007), H.1, S.51-59.

${ }^{24}$ John O'Loughlin, Chicago an der Ruhr Or What? Explaining the Location of Immigrants in European Cities, in: Günther Glebe/John O’Loughlin (Hrsg.), Foreign Minorities in Continental European Cities, Wiesbaden 1987, S.52-70; William A.V. Clark/Anita I. Drever, Residential Mobility in a Constrained Housing Market: Implications for Ethnic Populations in Germany, in: Environment and Planning A 32 (2000), H.5, S.833-846; Karen Schönwälder/Janina Söhn, Immigrant Settlement Structures in Germany: General Patterns and Urban Levels of Concentration of Major Groups, in: Urban Studies 46 (2009), H.7, S. 1439-1460.

${ }^{25}$ Philip Sarre/Deborah Phillips/Richard Skellington, Ethnic Minority Housing: Explanations and Policies, Aldershot 1989; Ceri Peach, Does Britain Have Ghettos?, in: Transactions of the Institute of British Geographers 21 (1996), H.1, S.216-235; Deborah Phillips/Cathy Davis/Peter Ratcliffe, British Asian Narratives of Urban Space, in: ebenda 32 (2007), H.2, S.217-234.
} 
ihrer Ankunft in Deutschland, wie bereits erwähnt, der Auffassung, dass es sich um einen zeitlich befristeten Aufenthalt handeln würde. Aus diesem Grund sprachen sie ihrer Wohnsituation eine nur geringe Bedeutung zu und akzeptierten oft billige Unterkünfte von schlechter Qualität, um möglichst viel Geld in ihre Heimatländer überweisen zu können. Zweitens wurden die Behörden in Westdeutschland zu einem gewissen Grad davon überrascht, dass der Anwerbestopp von 1973 aufgrund von Familienzusammenführungen zu einem deutlichen Anstieg der ausländischen Bevölkerung und zu einem damit zusammenhängenden Wohnungsmangel führte. ${ }^{26}$

Dabei wichen die Muster in Bremen nicht von denen andernorts in der Bundesrepublik ab. Die Unternehmen, die ausländische Arbeitnehmer einstellten, mussten angemessene Unterkünfte zur Verfügung stellen. ${ }^{27}$ Für Bremen lassen sich zahllose Dokumente finden, die in aller Ausführlichkeit vom Kauf der Liegenschaften handeln, auf denen Baracken für „Gastarbeiter“ errichtet werden sollten. Sie verweisen auf die Baurichtlinien und diskutieren die Perspektiven, die Arbeitskräfte nicht zu isolieren, sondern ihnen die Möglichkeit zu bieten, sich in ihrer Wohngegend $\mathrm{zu}$ integrieren. ${ }^{28}$ Trotz dieser ernsthaften Versuche, einen gewissen Standard bei den Unterkünften für „Gastarbeiter“ in Bremen zu entwickeln, ergaben sich rasch Schwierigkeiten: Beschwerden wegen der Überbelegung, mangelndes Heizen, Schikanen, Bettenmangel, schlechtes Essen und unzureichende sanitäre Anlagen. ${ }^{29}$ Hinzu traten Isolation und Segregation - Aspekte, die in Bremen Anfang der 1970er Jahre viel öffentliches Interesse auf sich zogen und vor allem um den Einsatz eines Wohnschiffes für „Gastarbeiter“, die Casa Marina, kreisten. ${ }^{30}$

Viele der türkischen Zuwanderer in Bremen lebten, entsprechend der Situation in der Bundesrepublik insgesamt, in überfüllten Wohnunterkünften von schlechter Qualität. Einem Bericht des Senats aus dem Jahr 1981 zufolge wohnten 83 Prozent aller Zuwanderer, deren Haushalt aus mindestens fünf Personen bestand, auf einer Fläche von unter $80 \mathrm{~m}^{2}$. Das galt im Vergleich für nur 19 Prozent der einheimischen Bevölkerung. Die Situation stellte sich für die türkischen Familien dabei als noch kritischer heraus, lebten doch 79 Prozent auf einer Fläche von unter $60 \mathrm{~m}^{2}$ und 51,6 Prozent auf weniger als $40 \mathrm{~m}^{2}$. In diesem Bericht wird zudem hervorgehoben, dass nur 28 Prozent der Zuwanderer, die Anspruch auf eine Sozialwohnung hatten, diesen auch geltend machen konnten. ${ }^{31}$ Die Kommunalverwaltung wies darüber hinaus auf den Modernisierungsbedarf der Wohnunterkünfte

\footnotetext{
${ }^{26}$ Lutz Holzner, The Myth of Turkish Ghettos: A Geographic Case Study of West German Responses Towards a Foreign Minority, in: The Journal of Ethnic Studies 9 (1982), H.4, S.65-85; Jürgen Friedrichs, Ethnic Segregation in Cologne, Germany, 1984-94, in: Urban Studies 35 (1998), H.10, S.17451763 .

${ }^{27}$ Ulrich Herbert, A History of Foreign Labor in Germany, 1880-1980: Seasonal Workers, Forced Laborers, Guest Workers, Ann Arbor 1990, S.217f.; Panayi, Ethnic Minorities in Nineteenth and Twentieth Century Germany, S. $220 \mathrm{f}$.

${ }^{28}$ Staatsarchiv Bremen, 7,2121/1-713 Sammlung von Schriftgut zu Personalmarketing, Beschäftigung und Unterbringung von ausländischen Arbeitern, zur Stahlbauerschule und zu Auszubildenden und Lehrlingen 1944-1975; Staatsarchiv Bremen, 4,31/6-68 Gastarbeiterwohnungen an der Rablinghauser Landstraße in Rablinghausen 1970-1975; Staatsarchiv Bremen, 4,124/3-692 Unterbringung von Ausländern 1972-1979.

${ }^{29}$ Staatsarchiv Bremen, 4,130/4-250 Orts- und Wohnungshygiene - Wohnunterkünfte für ausländische Arbeiter 1962-1975; Staatsarchiv Bremen, 7,2121/1-712 Sammlung von Schriftgut zur Beschäftigung, Unterbringung und Lage der ausländischen Arbeiter auf dem Vulkan 1969-1981.

${ }^{30}$ Gastarbeiter - ab ins Getto. Behörden wollen 200 Portugiesen in menschenleerem Hafengebiet ansiedeln, in: Weser Kurier, 7.8.1973; DGB kritisiert den Kauf des Gastarbeiter-Wohnschiffs, in: Bremer Nachrichten, 9.10.1973; Staatsarchiv Bremen, 4,13/4-122-10-02/14 Betreuung ausländischer Arbeitnehmer 1963-1975.

${ }^{31}$ Der Senator für das Bauwesen, Wohnen in Bremen (Der Senator für das Bauwesen informiert, H.2), Bremen, Juni 1981, S.3f.
} 
hin. ${ }^{32}$ Sowohl der Aspekt der Sozialwohnungen als auch jener der Sanierung waren Gegenstand einer Reihe innovativer Maßnahmen und Konzepte in Bremen, die die Zuwandererbevölkerung der Stadt betrafen. Sie wurden gebündelt in der „Konzeption zur Integration der ausländischen Arbeitnehmer und ihrer Familienangehörigen im Lande Bremen "vom Juni $1979 .{ }^{33}$

Obwohl sich in Bremen keine Ghettoisierung wie in den USA beobachten ließ, gab es in einigen Stadtteilen einen hohen Anteil von Zuwandererfamilien. Bereits in den 1980er Jahren wurde der rasche Anstieg des Migrantenanteils, insbesondere von Türken, aufmerksam verfolgt. ${ }^{34}$ Auch die südasiatische Bevölkerung in Newcastle hat sich im Laufe der Zeit vermehrt in bestimmten Teilen der Stadt zusammengefunden, mitunter in überfüllten Behausungen mit schwierigen Wohnbedingungen. Dennoch zeigt sich ein inhärenter Unterschied zwischen Bremen und Newcastle: Während sich die südasiatische Bevölkerung in Newcastle zunächst vor allem daran orientierte, dass das jeweilige Stadtviertel erschwinglichen Wohnraum bot, wählte die türkische Bevölkerung in Bremen ihren Wohnsitz in den Stadtteilen, in denen sich die oben genannten Arbeitgeber befanden. Diejenigen, die zum Beispiel im Westen der Stadt lebten, waren häufig in der Hafengegend beschäftigt, diejenigen in den östlichen Stadtteilen arbeiteten in der Regel für Daimler-Benz oder den Elektronikhersteller Nordmende, diejenigen in Bremen-Nord hatten oft bei der Bremer Vulkan AG oder der Bremer Woll-Kämmerei AG Arbeit gefunden. ${ }^{35}$

Während in Newcastle also die Wohnlage im Vordergrund stand, bildete in Bremen der Standort der Industriebetriebe das strukturierende Element. Dies verband sich mit Unterschieden in beiden Städten hinsichtlich der Orientierung der Migrantenbevölkerungen am Erwerb von Hauseigentum. Hausbesitz und wohnräumliche Unabhängigkeit scheinen für südasiatische Migranten in Newcastle ein wesentliches Ziel gewesen zu sein, wie eine in den 1970er Jahren durchgeführte Studie und verfügbare Archivdokumente belegen. ${ }^{36}$ In Bremen dagegen entschieden sich türkische Einwanderer erst deutlich später für den Kauf von Immobilien, selbst unter Berücksichtigung der Tatsache, dass der Anteil an Hausbesitzern in Deutschland generell niedriger lag als in Großbritannien und Gesamteuropa. ${ }^{37}$ Interviews, die in Bremen durchgeführt wurden, unterstützen die von Amelie F. Constant, Rowan Roberts und Klaus F. Zimmermann entwickelte These, wonach der Besitz

\footnotetext{
${ }^{32}$ Modernisierungsmaßnahmen im Ortsteil Lindenhof mit dem Ziel, für deutsche und ausländische Mieter besseren Wohnraum zu schaffen, Bremische Bürgerschaft, Stadt, 11. Wahlperiode, 11.Sitzung, 7.12.1984.

${ }^{33}$ Der Senat der Freien Hansestadt Bremen, Konzeption zur Integration der ausländischen Arbeitnehmer und ihrer Familienangehörigen im Lande Bremen, Juni 1979.

${ }^{34}$ Hans-Ewald Schnurr/Wiebke Blume, Räumlich-strukturelle Analyse der Ausländerkonzentration in der Stadt Bremen, in: Statistische Monatsberichte (Freie Hansestadt Bremen) 34 (1982), H.10, S.309327; Kinder im Vorschulalter und Ausländeranteile in der Stadt Bremen im Januar 1993 nach Stadtteilen, in: ebenda 46 (1994), H.2/3, S.36f.

${ }^{35}$ Hans-Ewald Schnurr, Die Segregation der ausländischen Bevölkerung in Bremen, in: Statistische Monatsberichte (Freie Hansestadt Bremen) 35 (1983), H.10, S.245-252; ders., Ausländer nach Ortsteilen der Stadt Bremen, in: ebenda 37 (1985), H.7, S.146-148.

${ }^{36}$ Davies, The Evangelistic Bureaucrat; Taylor, The Half-Way Generation; Hackett, Peering Around the ,Velvet Curtain of Culture‘. Die einzige Ausnahme unter der südasiatischen Bevölkerung in Newcastle bildeten die Bengalen. Siehe TWAS, MD.NC/162/1, Local Government and Racial Equality Sub-Committee of Corporate Joint Sub-Committee, 18.3.1983-17.7.1985; Director of Housing. Housing the Bengali Community in the Inner West End, 24.5.1984; Director of Environmental Health. The Bengali Community in the Inner West End of Newcastle, 31. 5.1984.

${ }^{37}$ Franz-Josef Kemper, Restructuring of Housing and Ethnic Segregation: Recent Developments in Berlin, in: Urban Studies 35 (1998), H.10, S.1765-1789, hier S.1769; Schönwälder/Söhn, Immigrant Settlement Structures in Germany, S. 1451.
} 
von Wohnungen Indikator für eine Bindung an das Aufnahmeland ist. ${ }^{38}$ Noch aktueller sind die Erkenntnisse von Sule Özüekren und Ebru Ergoz-Karahan, die davon sprechen, dass mit der Entscheidung für eine dauerhafte Niederlassung seit den 1980er Jahren ein Anstieg der Zahl von Hausbesitzern türkischer Herkunft in Deutschland zu verbuchen ist. Sie stellen außerdem die These auf, dass sich Hausbesitz und Unternehmensgründung parallel entwickelten. ${ }^{39}$

Viele der Befragten hatten sich erst in den 1990er Jahren zum Kauf eines Hauses entschlossen, für einige lag die Umsetzung dieses Ziels zum Zeitpunkt des Interviews noch in der Zukunft. ${ }^{40}$ Die späte Orientierung am Immobilienbesitz verhinderte nicht die Entstehung von ethnisch geprägten Straßen und Stadtteilen in Bremen. Wie auch in Newcastle findet sich in bestimmten Stadtteilen inzwischen eine Vielzahl an Moscheen, ethnischen Lebensmittelgeschäften sowie durch Einwanderer geprägte Jugendzentren. Bestimmte Gebiete in beiden Städten, die anfangs entweder aus finanziellen Gründen oder aufgrund der Nähe zum Arbeitsplatz für Zuwanderer besonders attraktiv waren, entwickelten durch die Herausbildung einer spezifischen ethnischen Identität weiterhin eine hohe Anziehungskraft. ${ }^{41}$ Der Hauptunterschied zwischen Newcastle und Bremen liegt darin, dass sich diese wohnräumlichen Muster in Bremen, bedingt durch die anfänglichen Einschränkungen des „Gastarbeiter“-Systems, erst deutlich später herausgebildet haben.

\section{Bildung}

Bei der Betrachtung der Integrationssituation hat der Bildungsbereich in Deutschland sowohl auf politischer als auch auf akademischer Ebene seit den 1960er Jahren die meiste Aufmerksamkeit auf sich gezogen. ${ }^{42}$ Die Forschungsliteratur betont, dass Schülerinnen und Schüler aus Migrantenfamilien sich vermehrt in den am wenigsten angesehenen Schulen wiederfinden, beim Übergang in das System der beruflichen Ausbildung benachteiligt werden und viele von ihnen es nicht schaffen, einen Schulabschluss zu erlangen. ${ }^{43}$

\footnotetext{
${ }^{38}$ Amelie F. Constant/Rowan Roberts/Klaus F. Zimmermann, Ethnic Identity and Immigrant Homeownership, in: Urban Studies 46 (2009), H.9, S. 1879-1898.

${ }^{39}$ Sule Özüekren/Ebru Ergoz-Karahan, Housing Experiences of Turkish (Im)migrants in Berlin and Istanbul: Internal Differentiation and Segregation, in: Journal of Ethnic and Migration Studies 36 (2010), H.2, S.257-274, hier S.266.

${ }^{40}$ Zwischen Januar 2006 und August 2010 wurden Interviews mit Mitgliedern der türkischen Gemeinschaft in Bremen durchgeführt.

${ }^{41}$ Mit der Art und Weise, in der türkische Gemeinschaften in bestimmten Gebieten ein Zugehörigkeitsgefühl schaffen, beschäftigen sich: Ayse Çă̆lar, Constraining Metaphors and the Transnationalisation of Spaces in Berlin, in: Journal of Ethnic and Migration Studies 27 (2001), H.4, S.601-613; Patricia Ehrkamp, Placing Identities: Transnational Practices and Local Attachments of Turkish Immigrants in Germany, in: ebenda 31 (2005), H. 2, S. 345-364.

${ }^{42}$ Dies zeigt sich in Bremen im Umfang der amtlichen Korrespondenz zu diesem Thema seit den 1960er Jahren. Siehe zum Beispiel: Staatsarchiv Bremen, 4,111/5-2277 Beschulung der Kinder ausländischer Arbeitnehmer - Regelung in Bremen: Durchführung (Grundsatzfragen, Einzelfälle) 19621971; Staatsarchiv Bremen, 4,111/5-2268 Beschulung der Kinder ausländischer Arbeitnehmer - KMKRundschreiben (Mitteilungen, Beschlüsse), Bd.1, 1964-1971; Staatsarchiv Bremen, 4,124/3-5 Planung in Bremen - Konzeption, Bd. 2, 1986.

${ }^{43}$ Thomas Faist, From School to Work: Public Policy and Underclass Formation among Young Turks in Germany during the 1980s, in: International Migration Review 27 (1993), H.2, S.306-331; Regina T. Riphahn, Cohort Effects in the Educational Attainment of Second Generation Immigrants in Germany: An Analysis of Census Data, in: Journal of Population Economics 16 (2003), H.4, S.711-737; Janina Söhn/Veysel Özcan, The Educational Attainment of Turkish Migrants in Germany, in: Turkish Studies 7 (2006), H.1, S.101-124.
} 
Anders als bei den Themen Arbeit und Wohnen konnten die Hauptunterschiede im Bildungsbereich im Hinblick auf die Jugendlichen aus Zuwandererfamilien in Bremen und Newcastle auf der Ebene der integrationspolitischen Konzepte und Maßnahmen, nicht aber bei den Leistungen oder dem Handeln der Zuwanderer selbst beobachtet werden. Für die deutsche Situation besteht insofern Konsens, als darauf verwiesen wird, dass Staat und Kommunen sehr langsam auf den Anstieg der Zahl von Schülerinnen und Schülern aus Zuwandererfamilien reagierten. Weil der Aufenthalt von „Gastarbeitern“ in Westdeutschland ursprünglich nur befristet sein sollte und nicht absehbar war, dass das Bildungssystem von der Zuwanderung betroffen sein würde, ist das nicht überraschend. Die Situation in Deutschland erweist sich auch deshalb als komplex, weil der Bildungsbereich der Länderhoheit obliegt und jedes Bundesland jene bildungspolitischen Maßnahmen durchsetzen kann, die es für angemessen erachtet. Die beiden deutschen Bundesländer, deren Regelungen im Bildungssystem gegenüber Schülern aus Zuwandererfamilien oft als gegensätzlich angesehen werden, sind Bayern und Berlin. In den 1970er Jahren setzte Bayern seinen Schwerpunkt auf das Erlernen der Muttersprachen und betonte vornehmlich die Probleme der Integration. Berlin unternahm im gleichen Zeitraum den Versuch, die Schüler auf eine Zukunft in Deutschland vorzubereiten. Andere Bundesländer kombinierten wiederum beide Modelle und setzten sowohl auf die Förderung des Deutschen als auch der Muttersprachen. ${ }^{44}$

Der bildungspolitische Ansatz des Landes Bremen lag jenem Berlins näher. Hauptziel war es, die Schüler in die deutsche Gesellschaft zu integrieren. In der zweiten Hälfte der 1970er Jahre führte das Land Bremen beispielsweise eine Reihe von Maßnahmen durch, um das Ziel der Integration zu erreichen. ${ }^{45}$ Dazu gehörten die Werbung für Kindertagesstätten bei Einwandererfamilien oder zusätzlicher Deutschunterricht. Es gab allerdings auch einige Maßnahmen, die den Zweck verfolgten, die Kinder auf eine mögliche Rückkehr in die Heimatländer vorzubereiten. Sie zielten vor allem darauf, die Kenntnisse über Muttersprachen und Herkunftskulturen zu vertiefen. Damit stand Bremen nicht allein, wie die Beispiele Nordrhein-Westfalen, Niedersachsen und Baden-Württemberg zeigen. ${ }^{46}$

Im Laufe der Zeit konzentrierten sich die politischen Maßnahmen in Bremen immer weiter auf die Integration von Schülern aus Einwandererfamilien in die deutsche Gesellschaft. Einem Bericht des Senats vom September 1980 zufolge ${ }^{47}$ sollte vornehmlich danach gestrebt werden, die Möglichkeiten des Erwerbs der deutschen Sprache zu verbessern, um für die Jugendlichen aus Einwandererfamilien die Chancen zu erhöhen, einen qualifizierten Schulabschluss zu erlangen. ${ }^{48}$ Darüber hinaus wurde bestimmt, dass der Anteil an Schülerinnen und Schülern aus Migrantenfamilien in einer Schule oder in einer Klasse nicht

\footnotetext{
${ }^{44}$ Ray Rist, On the Education of Guest-Worker Children in Germany: A Comparative Study of Policies and Programs in Bavaria and Berlin, in: The School Review 87 (1979), H.3, S.242-268; Theodor Hanf, Education in a Cultural Lag: The Case of Germany, in: International Journal of Educational Research 35 (2001), H.3, S.255-268.

${ }^{45}$ Antwort des Senats zur kleinen Anfrage der Fraktion der FDP vom 1.3.1977. Kinder ausländischer Arbeitnehmer, Drucksache 9/484 (Drs. 9445), 25.4.1977, Bremische Bürgerschaft, Landtag, 9.Wahlperiode.

${ }^{46}$ Barry McLaughlin/Peter Graf, Bilingual Education in West Germany: Recent Developments, in: Comparative Education 21 (1985), H.3, S.247f.; Eric Beck, Language Rights and Turkish Children in Germany, in: Patterns of Prejudice 33 (1999), H.2, S.3-12, hier S.8.

${ }^{47}$ Bericht über die Lage der schulpflichtigen Kinder von Ausländern, Drucksache 10/300, 1.9.1980, Bremische Bürgerschaft, Landtag, 10. Wahlperiode.

${ }^{48}$ Richard D. Alba/Johann Handl/Walter Müller, Ethnische Ungleichheit im deutschen Bildungssystem, in: Kölner Zeitschrift für Soziologie und Sozialpsychologie 46 (1994), S.209-227; Susanne Worbs, The Second Generation in Germany: Between Schools and Labor Market, in: International Migration Review 37 (2003), H.4, S.1011-1038.
} 
über 20 Prozent liegen solle. Informationsbroschüren für die Eltern in deren Muttersprachen wurden verteilt, Anträge auf Kindergeld durften nur noch bewilligt werden, wenn nachgewiesen worden war, dass die Kinder der Schulpflicht nachgingen. Veränderungen betrafen auch die Schulen selbst. Dazu gehörten die zusätzliche Unterstützung von Schülern, deren Deutschkenntnisse nicht ausreichten, um am normalen Schulunterricht teilzunehmen, sowie die Einführung von Vorbereitungsklassen mit dem Ziel, eine langfristige Integration zu fördern. Im Fokus stand zudem das Bemühen, den Anteil der Schülerinnen und Schüler aus Einwandererfamilien an Gymnasien zu erhöhen. ${ }^{49}$

Die städtischen Behörden in Newcastle folgten ganz anderen bildungspolitischen Prämissen: Sie orientierten sich sowohl an den Bedürfnissen temporärer Arbeitsmigration als auch dauerhafter Einwanderung. Zudem blieben die Maßnahmen im Ordnungsrahmen nationaler Richtlinien. Dies wurde erstmals in den 1960er Jahren deutlich, als die Stadt einen auf strikte Anpassung gerichteten Ansatz vertrat: Der Schwerpunkt lag auf dem Erwerb des Englischen, in Erwägung gezogen wurde die Durchsetzung eines Verteilungsschlüssels, der sicherstellen sollte, dass der Migrantenanteil in den einzelnen Schulen und Klassenräumen nicht über einem Drittel lag. Derartige Maßnahmen setzten in den 1960er und 1970er Jahren viele andere britische Städte ebenfalls durch. ${ }^{50}$ In den 1980er Jahren wurde dieser auf strikte Anpassung gerichtete Ansatz sowohl auf nationaler Ebene als auch in Newcastle durch einen multikulturellen Ansatz abgelöst. Insbesondere die bildungspolitischen Maßnahmen der zweiten Hälfte der 1980er Jahre beeinflusste der 1985 veröffentlichte Swann-Report, der auch unter dem Titel „Education for All“ bekannt ist. Der Bericht vertrat die Ansicht, dass Schülerinnen und Schüler in einem multikulturellen Umfeld unterrichtet werden sollten und die Trennung von einheimischen und zugewanderten Kindern und Jugendlichen schädlich sei. ${ }^{51}$ In Newcastle führte dieses Umdenken zu einer Förderung der Sprachen der Einwandererkinder sowie von muttersprachlichen Lehrern und zum Kampf gegen Rassismus in den Schulen. ${ }^{52}$ Auch in diesem Falle orientierte sich Newcastle an den Maßnahmen und Richtlinien, die andernorts in Großbritannien eingeführt wurden. ${ }^{53}$

\footnotetext{
${ }^{49}$ Joachim Frick/Gert Wagner, Economic and Social Perspectives of Immigrant Children in Germany, in: Edda Currle/Tanja Wunderlich (Hrsg.), Deutschland, ein Einwanderungsland? Rückblick, Bilanz und neue Fragen, Stuttgart 2001, S.299-326; Cornelia Kristen/Nadia Granato, The Educational Attainment of the Second Generation in Germany: Social Origins and Ethnic Inequality, in: Ethnicities 7 (2007), H.3, S.343-366.

${ }^{50} \mathrm{Zu}$ Newcastle siehe TWAS, MD.NC/149, Commonwealth Immigrants Working Group of Planning Committee, 19.9.1966-6.5. 1968. Immigrant Pupils in Schools, 5. 12.1967. Zu den politischen Maßnahmen anderer Städte siehe Henry Miller, Race Relations and Schools in Great Britain, in: Phylon 27 (1966), H.3, S.247-267; George A. Male, Multicultural Education and Education Policy: The British Experience, in: Comparative Education Review 24 (1980), H.3, S.291-301.

${ }^{51}$ Terry S. Chivers, Race and Culture in Education. Issues Arising from the Swann Committee Report, Windsor 1987; Gajendra K. Verma, Diversity and Multicultural Education: Cross-cutting Issues and Concepts, in: Gajendra K. Verma/Christopher Bagley/Madan Jha (Hrsg.), International Perspectives on Educational Diversity and Inclusion. Studies from America, Europe and India, Abingdon 2007, S.21-30.

${ }^{52}$ TWAS, MD.NC/162/2, Local Government and Racial Equality Sub-Committee of corporate Joint Sub-Committee, 18.9.1985-15.7.1987, Mother-Tongue Teaching Report on the Schools Language Survey conducted in Newcastle-upon-Tyne Schools, 18.9.1985; The Swann Report, 18.9.1985; Monitoring of Racial Incidents in Schools, 18.3.1987; Strategies for Combating Racism - Head Teachers' Courses, 18.3.1987.

${ }^{53}$ Gajendra K. Verma/Douglas Darby, Immigrant Policies and the Education of Immigrants in Britain, in: Pirkko Pitkänen/Devorah Kalekin-Fishman/Gajendra K. Verma (Hrsg.), Education and Immigration: Settlement Policies and Current Challenges, London 2002, S.11-47; Humayun Ansari, „The Infidel Within“: Muslims in Britain since 1800, London 2004, S.318-320.
} 
Sowohl in Newcastle als auch in Bremen wurde mithin seit den 1960er Jahren eine Vielzahl an Maßnahmen zur Integration durch Bildung durchgeführt. Während zu Beginn in Bremen, dem bundesdeutschen Trend entsprechend, die Teilhabe von Kindern der „Gastarbeiter“ am Bildungssystem für ein untergeordnetes Problem gehalten wurde, stellte sich die Kommunalverwaltung in Newcastle von Anfang an auf eine dauerhafte Niederlassung und Integration der südasiatischen Schüler ein. Die Frage, wie auf die steigende Zahl der türkischen Schülerinnen und Schüler der zweiten Generation bildungspolitisch zu reagieren sei, überforderte die Bremer Kommunalpolitik zunächst. ${ }^{54}$ Daraus ergaben sich erheblich größere Probleme als in anderen Bereichen; denn während türkische Einwanderer in Bremen und im gesamten Bundesgebiet die Möglichkeit hatten, ihre eigenen Arbeits- und Wohnungsmuster herauszubilden, waren türkische Schulkinder ohne größere eigene Handlungsspielräume einer vorgegebenen Palette an bildungspolitischen Richtlinien und Maßnahmen ausgesetzt.

Die Bedeutung des Bildungsbereichs in Bremen lässt sich auch aus der enormen kommunalpolitischen Aufmerksamkeit in den vergangenen Jahrzehnten ablesen. Interviews zeigten, dass viele der Migranten das deutsche Bildungssystem zwar nicht gänzlich verstanden hatten, die türkische Bevölkerung in Bremen jedoch sehr eindringlich die essentielle Bedeutung von Bildung für den Erfolg und die Integration ihrer Kinder betonte. ${ }^{55}$ Die Bildung von Kindern aus ethnischen Minderheiten in Deutschland hat durch die PISAStudien unlängst auch auf internationaler Ebene Aufmerksamkeit auf sich gezogen. Deutschland schnitt, sowohl in Bezug auf deutsche Kinder als auch auf Nachkommen der Zuwandererfamilien, insgesamt deutlich unter dem OECD-Durchschnitt ab, wodurch eine hitzige politische und wissenschaftliche Debatte in Gang gesetzt wurde ${ }^{56}$ Bezüglich Bremen sind die beiden Studien aus den Jahren 2000 und 2003 zu dem Schluss gekommen, dass Einwandererkinder ihren deutschen Mitschülern im Bereich der Lesekompetenz, der mathematischen Kenntnisse und des Gebrauchs der deutschen Sprache hinterherhinken. ${ }^{57}$ Unter den Gründen für das schlechte Abschneiden Deutschlands sollte das verspätete Erfüllen der Bedürfnisse von Schülerinnen und Schülern aus Einwandererfamilien nicht außer Acht gelassen werden. ${ }^{58}$

\footnotetext{
${ }^{54} \mathrm{Zu}$ Angaben bezüglich der wachsenden Anzahl an Schülern aus Einwandererfamilien in Bremen siehe Statistisches Landesamt Bremen, Schulbesuch und Schulerfolg ausländischer Schüler (Statistische Monatsberichte der Freien Hansestadt Bremen, 30 [April 1978]) und Staatsarchiv Bremen, 4,111/ 5-2398 Allgemeine Schulstatistik, vor allem öffentliche berufliche Schulen, private und staatliche berufliche Schulen, Schulen des Gesundheitswesens: Klassenfrequenzen, Klassen- und Schülerzahlen, Ausländeranteil; Absolventenprognose der allgemeinen öffentlichen und privaten Schulen, Schülerzahlprognosen, Klassenfrequenzen, Bd.1, 1989-1990.

${ }^{55}$ Zwischen Januar 2006 und August 2010 wurden Interviews mit Mitgliedern der türkischen Gemeinschaft in Bremen durchgeführt.

${ }^{56}$ Ammermüller, Poor Background or Low Returns?; Manuel Siegert, Schulische Bildung von Migranten in Deutschland, Nürnberg 2008.

${ }^{57}$ Manfred Prenzel/Jürgen Baumert/Werner Blum/Rainer Lehmann/Detlev Leutner/Michael Neubrand u.a., PISA 2003: Der zweite Vergleich der Länder in Deutschland - Was wissen und können Jugendliche?, Münster 2005, S.267-298.

${ }^{58}$ Cornelia Kristen, Ethnic Differences in Educational Placement: The Transition from Primary to Secondary Schooling (Mannheimer Zentrum für Europäische Sozialforschung [MZES], Arbeitspapiere, 32 [2000]); Sylke Viola Schnepf, Immigrants' Educational Disadvantage: An Examination Across Ten Countries and Three Surveys, in: Journal of Population Economics 21 (2007), H.3, S.527-545.
} 


\section{Fazit}

Die Literatur hat in Bezug auf Deutschland hervorgehoben, dass die Integration der Zuwanderer verzögert worden sei, weil die langfristigen Folgen der nur als temporär vorgestellten Zuwanderungen nicht erkannt worden seien. ${ }^{59}$ Der Fall Bremen verdeutlicht, dass die Erfahrungen von türkischen Zuwanderern tatsächlich erheblich durch die Nachwirkungen des „Gastarbeiter“-Systems beeinflusst und geprägt wurden. Im Vergleich zu Newcastle sind Segregation und Isolierung im Arbeits- und Wohnungsbereich erst spät erkannt und als politische Aufgabe verstanden worden. Im Bildungsbereich sorgte die Unsicherheit über die Zukunft türkischer Familien in einem Deutschland, das sich nicht als Einwanderungsland verstand, für Verunsicherungen und Hemmnisse bei der Frage, welche Richtlinien am geeignetsten und dringlichsten seien.

Dies bedeutet jedoch nicht, dass die lokalen Behörden in Bremen die wachsende türkische Migrantenbevölkerung nicht wahrnahmen und aus diesem Grund keinen Versuch unternahmen, sie angemessen zu unterstützen und zu versorgen. Tatsächlich belegen verfügbare Archivdokumente genau das Gegenteil; es wurde seit den 1960er Jahren ein klarer Schwerpunkt auf die Integration der „Gastarbeiter“ in Bremen gelegt. Die Dokumente beziehen sich auf den Arbeits-, Wohnungs- und Bildungsbereich sowie auf Aspekte wie Sprache und gesellschaftliche Beziehungen. Insgesamt gibt es Hinweise darauf, dass die Integration eindeutig als ein dringliches politisches Thema wahrgenommen wurde. ${ }^{60}$ Auch gab es ein Bewusstsein für die Situation der "Gastarbeiter“ im Bundesgebiet insgesamt und dafür, auf welche Weise andere Städte und Bundesländer die Integration als politische und gesellschaftliche Aufgabe angingen. ${ }^{61}$ Die Presse, wie auch die vielfältigen gesellschaftlichen Initiativen, die in dem Versuch gestartet wurden, Integration zu fördern, spielten dabei eine bedeutende Rolle. ${ }^{62}$ Es kam sogar so weit, dass die Bremer Vorgehensweise in ganz Deutschland Anerkennung fand. ${ }^{63}$

\footnotetext{
${ }^{59}$ Herbert, A History of Foreign Labor in Germany; Stephen Castles, Migrants and Minorities in PostKeynesian Capitalism: The German Case, in: Malcolm Cross (Hrsg.), Ethnic Minorities and Industrial Change in Europe and North America, Cambridge 1992, S.36-54; Joppke, Immigration and the NationState.

${ }^{60}$ Siehe zum Beispiel Staatsarchiv Bremen, 4,111/5-253 Durchführung des Unterrichts für Kinder ausländischer Arbeitnehmer - Berichte, Meldungen und Anfragen der Schule Schmidtstraße 19691976; Staatsarchiv Bremen, 7,2121/1-712 Sammlung von Schriftgut zur Beschäftigung, Unterbringung und Lage der ausländischen Arbeiter auf dem Vulkan 1969-1981; Staatsarchiv Bremen, 4,124/ 3-644 Sozialhilfe für Ausländer und Staatenlose - Allgemeines, Bd.2a 1973; Staatsarchiv Bremen, 4,124/3-650 Arbeitsgemeinschaft ausländischer Arbeiter (AGaA) 1974; Staatsarchiv Bremen, 4,124/ 3-649 Sozialhilfe für Ausländer und Staatenlose - Allgemeines, Bd.6, 1979-1980; Staatsarchiv Bremen, 4,111/7-254 Studienkolleg Bremen - Sonderkurs für Gastarbeiterkinder 1979-1983.

${ }^{61}$ Staatsarchiv Bremen, 4,124/3-694 Ausländische Arbeitskräfte in Deutschland, Bd. 1, 1960-1963; Staatsarchiv Bremen 4,111/5-2290 Beschulung der Kinder ausländischer Arbeitnehmer - Allgemeines (1955), Bd.1, 1964-1971; Staatsarchiv Bremen, 4,124/3-697 Ausländische Arbeitskräfte in Deutschland, Bd.4, 1971-1974; Staatsarchiv Bremen 4,124/3-9 Außerschulische Jugendarbeit, Bd.1, 1979-1981; Staatsarchiv Bremen, 4,124/3-5 Planung in Bremen - Konzeption, Bd.2, 1986.

${ }^{62} 14000$ Gastarbeiter in Bremen. Die Quote liegt weiter unter Bundesdurchschnitt - Integration mit relativ wenig Schwierigkeiten, in: Bremer Bürgerzeitung, 24.9.1971; „Gleiche Rechte einräumen“. Bürgerschaft fordert Programm zur Integration der Gastarbeiter, in: Bremer Nachrichten, 5.10.1973; Staatsarchiv Bremen, 4,124/3-650 Arbeitsgemeinschaft ausländischer Arbeiter (AGaA) 1974; Staatsarchiv Bremen, 4,124/3-22 Neustrukturierung der Sozialarbeit für ausländische Familien, Bd.3, 1981.

${ }^{63}$ Bremen will Gastarbeiterkindern besseren Unterricht anbieten, in: Frankfurter Rundschau, 11.4.1978; Die soziale Zeitbombe tickt nicht überall. Bremens Schule an der Schmidtstraße als Modell für die Integration, in: Stuttgarter Zeitung, 21.9.1979.
} 
Anders als die Literatur über westdeutsche Integrationspolitik insgesamt zu vermitteln sucht, hat sich Bremen in den 1960er und 1970er Jahren nicht blauäugig auf das Thema Immigration eingelassen. Trotz allem gibt es keinen Zweifel daran, dass das „Gastarbeiter"-System und seine Nachwirkungen einen langfristigen Einfluss auf das Handeln und die Erfahrungen der türkischen Zuwandererbevölkerung in Bremen hatten. Als zentral erwies sich dabei, dass die integrationspolitischen Bemühungen des Bremer Senats in den 1960er und 1970er Jahren innerhalb des einschränkenden Handlungsrahmens des „Gastarbeiter"-Systems unternommen wurden. Während die Kommunalverwaltung in Newcastle die dauerhafte Niederlassung der südasiatischen Einwanderer als gegeben ansah, mussten die Initiativen in Bremen scheitern, weil die Zuwanderung der „Gastarbeiter“ für ein temporäres Phänomen gehalten wurde. Allerdings hat Bremen weitaus früher als andere Bundesländer die Einwanderungssituation anerkannt. 
